\title{
Radio Wave Heating of Astrophysical Plasmas
}

\author{
R. T. Gangadhara* \& V. Krishan Indian Institute of Astrophysics, Bangalore \\ 560034
}

Received 1990 February 16; revised 1990 August 1; accepted 1990 October 15

\begin{abstract}
The coherent plasma process such as parametric decay instability (PDI) has been applied to a homogeneous and unmagnetized plasma. These instabilities cause anomalous absorption of strong electromagnetic radiation under specific conditions of energy and momentum conservation and thus cause anomalous heating of the plasma. The maximum plasma temperatures reached are functions of luminosity of the radio radiation and plasma parameters. We believe that these processes may be taking place in many astrophysical objects. Here, the conditions in the sources 3C 273, 3C 48 and Crab Nebula are shown to be conducive to the excitation of PDI. These processes also contribute towards the absorption of $21 \mathrm{~cm}$ radiation.
\end{abstract}

Key words: plasma-parametric instability-quasar-Crab Nebulaanomalous absorption-electromagnetic waves

\section{Introduction}

One of the fundamental processes in the interaction of intense electromagnetic (e.m.) radiation, of frequency close to plasma frequency, with fully ionized plasmas is the parametric excitation of two new waves. If both the excited modes are purely electrostatic, they are eventually absorbed in the plasma due to collisional and Landau (wave-particle interaction) dampings. Landau damping of excited electrostatic waves occurs when their phase velocity is slightly larger than the thermal velocity of the plasma particles. When the wave dissipates, its energy gets converted into thermal energy of particles. The incident e.m. radiation is anomalously absorbed in the plasma at a rate which is much larger than the collisional absorption rate.

Quasars, Seyfert galaxies and pulsars are among the most luminous objects in the universe, emitting strongly at radio frequencies. Here we study PDI in 3C 273, 3C 48 and the Crab Nebula.

\subsection{Density and Temperature Structure of Broad-line Regions of Active Galactic Nuclei}

The emission line regions of active galactic nuclei (AGN) are commonly separated into a "narrow-line region" (NLR) and a "broad-line region" (BLR). These regions consist

* Joint Astronomy programme, Department of Physics, Indian Institute of Science, Bangalore 560012 
of photoionized "clouds" moving with large velocities, usually assumed to be embedded in a hot dilute medium providing confinement. It has been recognized for a long time that the intense thermal and/or nonthermal UV and X-ray radiation emitted by AGN is responsible for heating and ionizing the line-emitting regions. The density structure of the BLR is closely related to the ionization structure (Kwan \& Krolik 1981). The density deduced in the models has gradually increased from $10^{7} \mathrm{~cm}^{-3}$ up to $10^{10} \mathrm{~cm}^{-3}$, a value which is now commonly assumed. The absence of broad components in the forbidden lines is interpreted as due to collisional deexcitation, so that the electron density must be greater than $10^{7} \mathrm{~cm}^{-3}$. On the other hand the presence of the broad semiforbidden C III] $\lambda 1909$ line which starts being deexcited at $n_{\mathrm{e}}>10^{9} \mathrm{~cm}^{-3}$ implies $n_{\mathrm{e}}<10^{10} \mathrm{~cm}^{-3}$. Larger densities are however, indicated by some line intensities like those of Fe II. The high ionization zone (H II), facing the central source of ionizing radiation, emits what we call the high ionization lines (HIL) $\mathrm{L}_{\alpha}, \mathrm{N} v$, $\mathrm{C}$ IV, He II, C III], etc. The temperature of the H II zone varies between about $15000 \mathrm{~K}$ and $25000 \mathrm{~K}$ according to the values of the density and the ionization parameter. The BLR has a dimension of 0.01-1 parsec, and clouds have electron densities $10^{9}-10^{10} \mathrm{~cm}^{-3}$ and temperatures $1.5 \times 10^{4}-2.5 \times 10^{4} \mathrm{~K}$ (Collin-Souffrin \& Lasota 1988). The BLR is very close to the active nucleus and therefore is an important diagnostic of the central region of AGN.

Davidson \& Netzer (1979) have reviewed some of the heating processes proposed by several workers. Here, we propose one more process for the absorption of radio waves and hence the heating of line-emitting regions: collective plasma process such as PDI driven by intense radio radiation emitted from AGN.

\subsection{The Radio Source}

Most of the radio emission in the quasars is assumed to be emitted from the accretion disc and also from the region interior to the BLR. The radio flux $f_{v}$ of $3 \mathrm{C} 48$ at $1400 \mathrm{MHz}$ is 15.63 Jansky and that of 3C 273 is 46.4 Jansky (Lang 1980). The redshift of 3C 48 is $z=0.367$ and that of 3C 273 is $z=0.158$. We find the distance $d$ between the observer and quasar using the Hubble distance-redshift relation $d=5000 \mathrm{Mpc}$. The luminosity of 3C 48 and that of 3C 273 at $1400 \mathrm{MHz}$, determined by using the relation $L_{v}=4 \pi d^{2} f_{v}$, are $5.95 \times 10^{34} \mathrm{erg} \mathrm{s}^{-1} \mathrm{~Hz}^{-1}$ and $3.28 \times 10^{34} \mathrm{ergs}^{-1} \mathrm{~Hz}^{-1}$, respectively. The total luminosity of Crab Nebula is $10^{38} \mathrm{erg} \mathrm{s}^{-1}$, twelve percent of which is in the radio band. The electron density and temperature at a distance of about 0.03 parsec from pulsar are about $10^{7} \mathrm{~cm}^{-3}$ and $10^{4} \mathrm{~K}$, respectively (Ginzburg \& Ozernoy 1966). The excitation of PDI in the emission-line regions of quasar was first discussed by Krishan (1987) and then by Gangadhara \& Krishan (1989, 1990). Here, we extend this work to include the new effects associated with strong and broad pump in the three astrophysical objects mentioned above.

\section{Parametric decay instability (PDI)}

An incident e.m. pump wave $\left(\omega_{0}, \mathbf{K}_{0}\right)$ excites a high frequency electron plasma wave $\left(\omega_{\mathrm{e}}, \mathrm{K}_{\mathrm{e}}\right)$ and a low frequency ion-acoustic wave $\left(\omega_{\mathrm{i}}, \mathbf{K}_{\mathrm{i}}\right)$ such that

$$
\omega_{0}=\omega_{\mathrm{e}}+\omega_{\mathrm{i}} \text { and } \quad \mathbf{K}_{0}=\mathbf{K}_{\mathrm{e}}+\mathbf{K}_{\mathrm{i}}
$$


where

$$
\omega_{0}^{2}=\omega_{\mathrm{pe}}^{2}+k_{0}^{2} c^{2}, \quad \omega_{\mathrm{e}}^{2}=\omega_{\mathrm{pe}}^{2}+\frac{3}{2} k^{2} v_{\mathrm{e}}^{2} \quad \text { and } \quad \omega_{\mathrm{i}}^{2}=\omega_{\mathrm{pi}}^{2}\left(1+\frac{1}{\left(k \lambda_{\mathrm{De}}\right)^{2}}\right)^{-1} .
$$

$\omega_{\mathrm{p} j}=\left[4 \pi \mathrm{n}_{j} e^{2} / m_{j}\right]^{1 / 2}, \quad v_{j}=\left[2 k_{\mathrm{B}} T_{j} / m_{j}\right]^{1 / 2}$ and $\quad \lambda_{\mathrm{D} j}=\left[k_{\mathrm{B}} T_{\mathrm{j}} / 4 \pi n_{j} e^{2}\right]^{1 / 2}$ are the plasma angular frequency, thermal velocity and Debye length of $j$ th species $(j=e, i)$, respectively. $k_{\mathrm{B}}$ and $c$ are the Boltzmann constant and velocity of light. The density, mass and temperature of $j$ th species are $n_{j}, m_{j}$ and $T_{j}$, respectively.

We chose $\omega_{0} \simeq \omega_{\mathrm{e}}, k_{0}^{2} c^{2} \ll \omega_{\mathrm{pe}}^{2}$ and obtain $K_{\mathrm{e}} \simeq-K_{\mathrm{i}}$. From equation (1), when $k \lambda_{D \mathrm{e}} \ll 1$, we find the wave number $k_{\mathrm{e}} \simeq k_{\mathrm{i}}=k$ of the excited Langmuir waves to be

$$
k=\lambda_{\mathrm{De}}^{-1}\left[\left\{\left(\frac{\omega_{\mathrm{pi}}}{\omega_{\mathrm{pe}}}\right)^{2}+\frac{2}{3}\left(\frac{\omega_{0}}{\omega_{\mathrm{pe}}}-1\right)\right\}^{1 / 2}-\frac{\omega_{\mathrm{pi}}}{3 \omega_{\mathrm{pe}}}\right] .
$$

The non-relativistic theory of parametric instabilities has been developed by Drake et al. (1974) for the case of linearly polarized pumps. The PDI can be described by using the dispersion relation (Liu \& Kaw 1976):

$$
\begin{aligned}
\frac{1}{\chi_{\mathrm{e}}(\overline{\mathbf{K}}, \omega)}+\frac{1}{\chi_{\mathrm{i}}(\mathbf{K}, \omega)+1}= & --\left(\mathbf{K} \cdot \mathbf{V}_{0}\right)^{2}\left[\frac{1}{\left(\omega-\omega_{0}\right)^{2} \varepsilon\left(\mathbf{K}, \omega-\omega_{0}\right)}\right. \\
& \left.+\frac{1}{\left(\omega+\omega_{0}\right)^{2} \varepsilon\left(\mathbf{K}, \omega+\omega_{0}\right)}\right]
\end{aligned}
$$

where $\mathrm{w}$ and $\mathbf{K}$ are the angular frequency and wave vector of the low frequency daughter mode. The quiver velocity of electrons in the pump electric field $\mathbf{E}_{0}$ is $v_{0}=e E_{0} / m_{\mathrm{e}} \omega_{0}$. The electron and ion susceptibilities are $\chi_{\mathrm{e}}$ and $\chi_{\mathrm{i}}$, respectively. The dielectric function of the plasma is $\varepsilon\left(\mathbf{K}, \omega \pm \omega_{0}\right)=1+\chi_{\mathrm{e}}\left(\mathbf{K}, \omega \pm \omega_{0}\right)+\chi_{\mathrm{j}}\left(\mathbf{K}, \omega+\omega_{0}\right)$.

We note that $\chi_{\mathrm{e}}$ and $\chi_{\mathrm{i}}$ have the following asymptotic forms:

$$
\begin{aligned}
& \chi_{\mathrm{e}}(\mathbf{K}, \omega)= \begin{cases}-\frac{\omega_{\mathrm{pe}}^{2}}{\omega^{2}}\left(1+\frac{3}{2} \frac{k^{2} v_{\mathrm{e}}^{2}}{\omega^{2}}\right)+i \frac{\sqrt{\pi}}{\left(k \lambda_{\mathrm{De}}\right)^{2}} \frac{\omega}{k v_{\mathrm{e}}} \exp \left\{-\omega^{2} /\left(k v_{\mathrm{e}}\right)^{2}\right\} & \text { for } \omega \gg k v_{\mathrm{e}} \\
\frac{1}{\left(k \lambda_{\mathrm{De}}\right)^{2}}\left(1+i \sqrt{\pi} \frac{\omega}{k v_{\mathrm{e}}}\right) & \text { for } \omega \ll k v_{\mathrm{e}}\end{cases} \\
& \chi_{\mathrm{i}}(\mathbf{K}, \omega)= \begin{cases}-\frac{\omega_{\mathrm{pi}}^{2}}{\omega^{2}}+i \frac{\sqrt{\pi}}{\left(k \lambda_{\mathrm{De}}\right)^{2}} \frac{T_{\mathrm{e}}}{T_{\mathrm{i}}} \frac{\omega}{k v_{\mathrm{i}}} \exp \left\{-\omega^{2} /\left(k v_{\mathrm{i}}\right)^{2}\right\} & \text { for } \omega \gg k v_{\mathrm{i}} \\
\frac{1}{\left(k \lambda_{\mathrm{De}}\right)^{2}} \frac{T_{\mathrm{e}}}{T_{\mathrm{i}}}\left(1+i \sqrt{\pi} \frac{\omega}{k v_{\mathrm{i}}}\right) & \text { for } \omega \ll k v_{\mathrm{i}}\end{cases}
\end{aligned}
$$

The energy density of the pump field $E_{0}$ and luminosity of the source are related by

$$
\frac{E_{0}^{2}}{8 \pi}=\frac{L}{4 \pi R^{2} c}
$$

where $L=L_{41} \times 10^{41} \mathrm{erg} \mathrm{s}^{-1}$ and $R=r_{\mathrm{pc}} \times 3.1 \times 10^{18} \mathrm{~cm}$ are the luminosity of the source and the distance between the source and plasma, respectively. Now, the 
oscillation velocity of electrons in the pump field is given by

$$
\begin{aligned}
v_{0} & =\frac{e E_{0}}{m_{\mathrm{e}} \omega_{0}} \\
& =\frac{e}{m_{\mathrm{e}}}\left(\frac{2 L}{R^{2} c}\right)^{1 / 2} \frac{1}{\omega_{0}} .
\end{aligned}
$$

If the pump is strong enough, the normal modes get modified and no more remain linear. An intense pump can cause amplitude dependent shift in the real and imaginary parts of the normal mode frequencies. For $\omega_{0}>\omega_{\mathrm{e}}$, PDI occurs due to the parametric decay of a pump wave into an electron and an ion mode. Depending on the pump strength and the ratio $T_{\mathrm{e}} / T_{\mathrm{i}}$, it can occur through three different routes: PDI-1, PDI-2, PDI-3, discussed below. However, when $\omega_{0}<\omega_{\mathrm{e}}$, a purely growing (zero frequency) ion density fluctuation will spontaneously grow from the noise level. As it grows in amplitude, so also does the associated electron plasma wave. This case of parametric instability is called the "oscillating two stream instability" (OTS).

\subsection{PDI-1: Decay of a Pump Wave into an Electron Plasma Wave and an Ion-Acoustic Wave $\left(\omega_{11}\right)$}

Here $\omega_{0} \simeq \omega_{\mathrm{e}}+\omega_{\mathrm{i} 1}$. For $k \lambda_{\mathrm{De}} \ll 1$ and $\omega_{\mathrm{pi}} \gg \omega_{\mathrm{i}}=k c_{\mathrm{s}}$, we neglect $\varepsilon\left(\mathbf{K}, \omega+\omega_{0}\right)$ as being off resonant. Using the asymptotic forms for $\chi_{\mathrm{e}}$ and $\chi_{\mathrm{i}}$ corresponding to $k v_{\mathrm{e}} \gg w \geqslant k v_{\mathrm{i}}$ Equation (3) becomes

$$
\left(\omega^{2}+i 2 \Gamma_{\mathrm{i}} \omega-k^{2} c_{\mathrm{s}}^{2}\right)\left(\omega-\Delta+i \Gamma_{\mathrm{e}}\right)+\frac{\left(\mathbf{K} \cdot \mathbf{V}_{\mathrm{o}}\right)^{2}}{2 \omega_{\mathrm{pe}}} \omega_{\mathrm{pi}}^{2}=0
$$

where $\Delta=\omega_{0}-\omega_{e}$ is the frequency mismatch. The ion-acoustic velocity is $c_{\mathrm{s}}=\left[{ }_{(k \mathrm{~B}} T_{\mathrm{e}}\right.$ $\left.\left.+3 k_{\mathrm{B}} T_{\mathrm{i}}\right) / m_{\mathrm{i}}\right]^{1 / 2}$. The damping rate of the electron plasma wave is the sum of the Landau damping rate and the electron collision frequency, given by

$$
\Gamma_{\mathrm{e}}=\frac{\sqrt{\pi}}{2} \frac{\omega_{\mathrm{pe}}}{\left(k \lambda_{\mathrm{De}}\right)^{3}} \exp \left\{-\frac{1}{2\left(K \lambda_{\mathrm{De}}\right)^{2}}-\frac{3}{2}\right\}+v_{\mathrm{e}} .
$$

The collisional damping rate of the electron plasma wave is $v_{\mathrm{e}}=3.632 n_{\mathrm{e}}, \ln \Lambda / T^{3 / 2}$ e. The Coulomb logarithm is In $\Lambda \simeq 10$. The damping rate of the ion-acoustic wave is the sum of the Landau damping rate and the ion collision frequency, given by

$$
\Gamma_{\mathrm{i}}=\left(\frac{\pi}{8}\right)^{1 / 2} k c_{\mathrm{s}}\left[\left(\frac{m_{\mathrm{e}}}{m_{\mathrm{i}}}\right)^{1 / 2}+\left(\frac{T_{\mathrm{e}}}{T_{\mathrm{i}}}\right)^{3 / 2} \exp \left\{-\frac{1}{2}\left(3+\frac{T_{\mathrm{e}}}{T_{\mathrm{i}}}\right)\right\}\right]+v_{\mathrm{i}}
$$

The collisional damping rate of the ion-acoustic wave is given by $v_{\mathrm{i}}$ $=0.0598 n_{\mathrm{i}} \ln \Lambda / T_{\mathrm{i}}^{3 / 2}$.

Like electrostatic waves, e.m. waves also experience collisional damping in the plasma medium. If $v_{0}$ is the collisional damping rate of e.m. wave, then the rate of energy loss from the e.m. wave $\left(v_{0} E_{0}^{2} / 8 \pi\right)$ must balance the rate at which the oscillatory energy of electrons is randomized by the electron-ion scattering, i.e. 
$\left(v_{0} E_{0}^{2} / 8 \pi\right)=\left(v_{\mathrm{e} i} m_{\mathrm{e}} v_{0}^{2} / 2\right)$. Since $v_{0}=e E_{0} / m_{\mathrm{e}} \omega_{0}$, this power balance gives

$$
v_{0}=\left(\frac{\omega_{\text {pe }}}{\omega_{0}}\right)^{2} v_{\text {ei }}
$$

where $v_{\mathrm{ei}} \simeq v_{e}$ is the electron-ion collision frequency. The dependence of $v_{0}$ on electron temperature $T_{\mathrm{e}}$ is shown in Figs 2(a), 3(a) and 4(a). Krolik, McKee \& Tarter (1978) have pointed out that the radio frequency waves can heat the plasma through free-free absorption process.

For $\omega=\omega_{11}=\omega_{\mathrm{r} 1}+i \gamma_{1}$, Equation (5) gives two coupled equations cubic in $\omega_{\mathrm{r} 1}$ and $\gamma_{1}$. Using $\omega_{\mathrm{r} 1}=k c_{\mathrm{s}}$ and $\gamma_{1} \omega_{\mathrm{rl}}$, we find only one root $\left(\omega_{\mathrm{r} 1}, \gamma_{1}\right)$ which represents growing mode while the other two roots represent damped modes because their growth rates are negative. The growth rate of growing mode is given by

$$
\gamma_{1}=-\frac{\left(\Gamma_{\mathrm{e}}+\Gamma_{\mathrm{i}}\right)}{2}+\operatorname{Re}\left[\frac{\left(\mathbf{K} \cdot \mathbf{V}_{0}\right)^{2}}{4 k c_{\mathrm{s}}}\left(\frac{m_{\mathrm{e}}}{m_{\mathrm{i}}}\right)^{1 / 2} \omega_{\mathrm{pi}}+\frac{\left(\Gamma_{\mathrm{i}}-\Gamma_{\mathrm{e}}-i \Delta\right)^{2}}{4}+i \frac{\Gamma_{\mathrm{e}} \Delta}{2}\right]^{1 / 2} \text {. }
$$

We assume $\mathrm{K} \| \mathrm{E}_{0}$, (the electric field of pump wave), $n_{\mathrm{i}}=n_{\mathrm{e}}$ in the hydrogen plasma and $\omega_{0}=1.01 \omega_{\text {pe }}$. Though we have chosen initially single frequency $\omega_{0}=1.01 \omega_{\text {pe }}$, as the instability sets up plasma temperatures $T_{\mathrm{e}}$ and $T_{\mathrm{i}}$ go up due to the damping of excited electrostatic waves and so do $\omega_{e}$ and $\omega_{\mathrm{i}}$. Since $\omega_{0}=\omega_{\mathrm{e}}+\omega_{\mathrm{i}}$, the resonant pump frequency should also increase. Therefore, a band of pump frequencies satisfies the resonance condition. An inhomogeneity in plasma density will also admit a broad pump. Therefore, we assume that the maximum band width $\Delta \omega \simeq \omega_{0}$ satisfies the resonant condition of the parametric decay instability. Since $\gamma_{1}$ is the growth rate due to a monochromatic pump at $\omega_{0}$, the actual growth rate $\gamma_{1}^{\prime}$ due to the broad pump with spectral width $\Delta \omega \gg \gamma_{1}$ is given by

$$
\gamma_{1}^{\prime}=\frac{1}{\Delta \omega} \gamma_{1}^{2}
$$

Hence due to a broad pump the growth rate of PDI is decreased by a factor of $\Delta \omega / \gamma_{1}$ (Kruer 1988). The coherence condition is satisfied for $\Delta \omega \leqslant \gamma_{1}$. Since the resonance condition for the instability is satisfied for the fraction of the band width, i.e., for $\Delta \omega \leqslant \gamma_{1}$, only a portion of the total luminosity is useful for the excitation of the instability.

The threshold condition obtained by setting $\gamma_{1}$ equal to zero in Equation (7), is given by

$$
\frac{\left(\mathbf{K} \cdot \mathbf{V}_{\mathrm{o}}\right)^{2}}{4 k c_{\mathrm{s}}} \omega_{\mathrm{pi}}\left(\frac{m_{\mathrm{e}}}{m_{\mathrm{i}}}\right)^{1 / 2}=\Gamma_{\mathrm{e}} \Gamma_{\mathrm{i}}
$$

The threshold is higher for an incoherent pump because incoherency effectively amounts to having more collisions in the system. A partially polarized pump again offers only a part of its luminosity for the excitation of PDI.

We introduce a decay parameter $D$, defined from Equation (8), as

$$
D=\frac{\int_{v_{1}}^{v_{2}} L_{v} d v}{4 \pi R^{2} n_{\mathrm{e}} c} P\left(\lambda_{\mathrm{De}}, k\right)
$$


where the function $P\left(\hat{\lambda}_{\text {De }}, k\right)=\frac{k \omega_{\mathrm{pi}}}{2 c_{\mathrm{s}}\left(m_{\mathrm{e}} m_{\mathrm{i}}\right)^{1 / 2}} \frac{1}{\Gamma_{\mathrm{e}} \Gamma_{\mathrm{i}}}$ expresses the ffect of plasma parameters on the instability. The integration over the luminosity has to be computed over the width $v_{2}-v_{1}=\Delta \omega / 2 \pi$. The variation of the function $P$ with respect to $T_{e}$ for different densities is shown in Fig. 1(a). The bending of curves at $K \lambda_{\mathrm{De}} \ll 1$ is due to collisional damping. But when $k \lambda_{\mathrm{De}} \simeq 0.4$ Landau damping operates which is a strong function of $T_{e}$ but not of $n_{\mathrm{e}}$. The strength of $P$ describes if the conditions in the plasma are favourable for PDI to occur. For small pump, PDI is favoured at large P. PDI occur for $D>1$ and switch off $D \leqslant 1$. for The variation of $D$ with respect to temperature $T_{\mathrm{e}}$ is shown in Fig. 1(b). When $D \gg 1$ the ionacoustic mode loses its linearity and the instability falls into one or the other three cases discussed below.

\subsection{Resonant Interaction Regions}

The average values of different parameters in the resonant interaction region of radio waves in the different sources, determined observationally, are listed in Table 1. Table 2 shows different values of ion temperature $T_{\mathrm{i}}$ and $\omega_{0}$ used for each case of PDI.

Electrons being lighter generally heat up before the ions and since the electron-ion collision time is much larger than the excitation time of PDI the inequality in temperature will remain during the instability.

We solve Equation (5) numerically for real and imaginary parts of $\omega$ and exhibit the results for PDI-1 in Figs 1(c) and 2(a). The region $\log T_{\mathrm{e}}>5.08$ in Fig. 1(c) for 3C 273

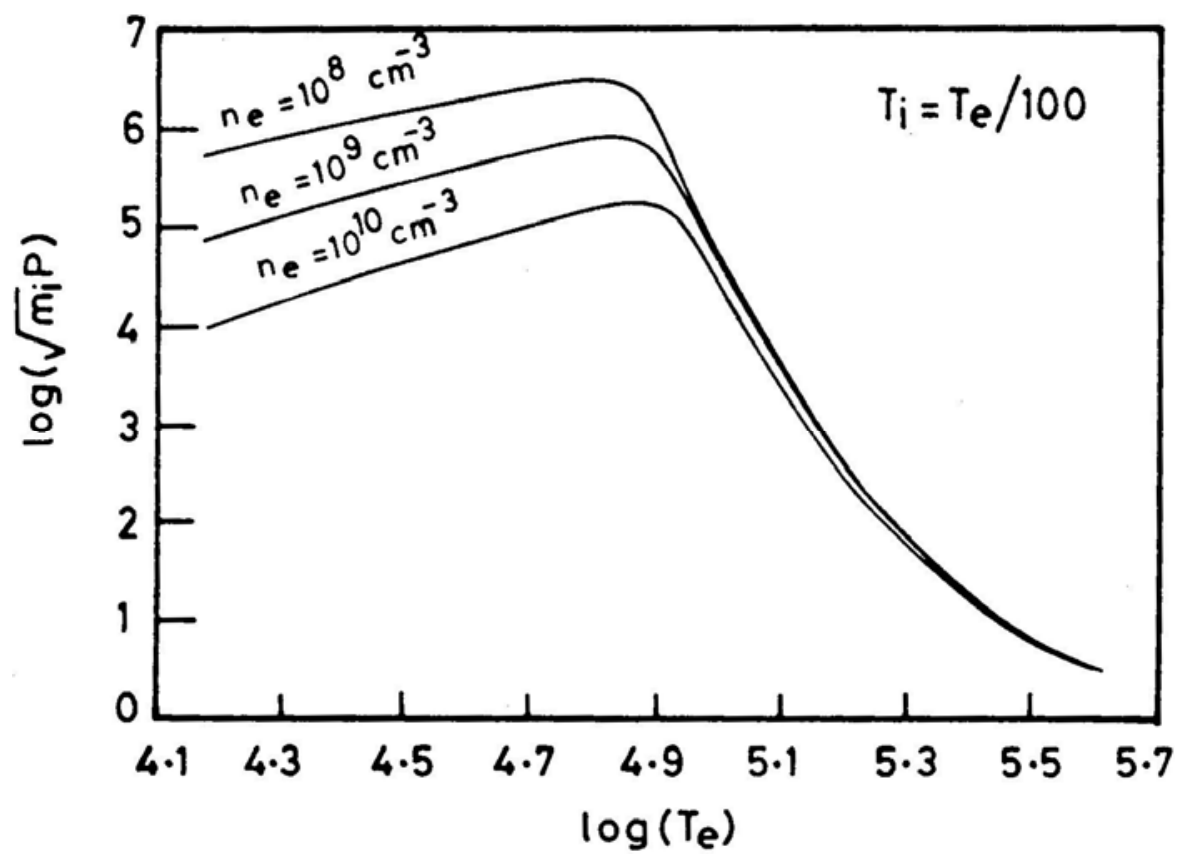

Figure 1. (a) Variation of $\sqrt{m_{\mathrm{i}}} P$ with electron temperature $T_{\mathrm{e}}$ for different electron densities. 


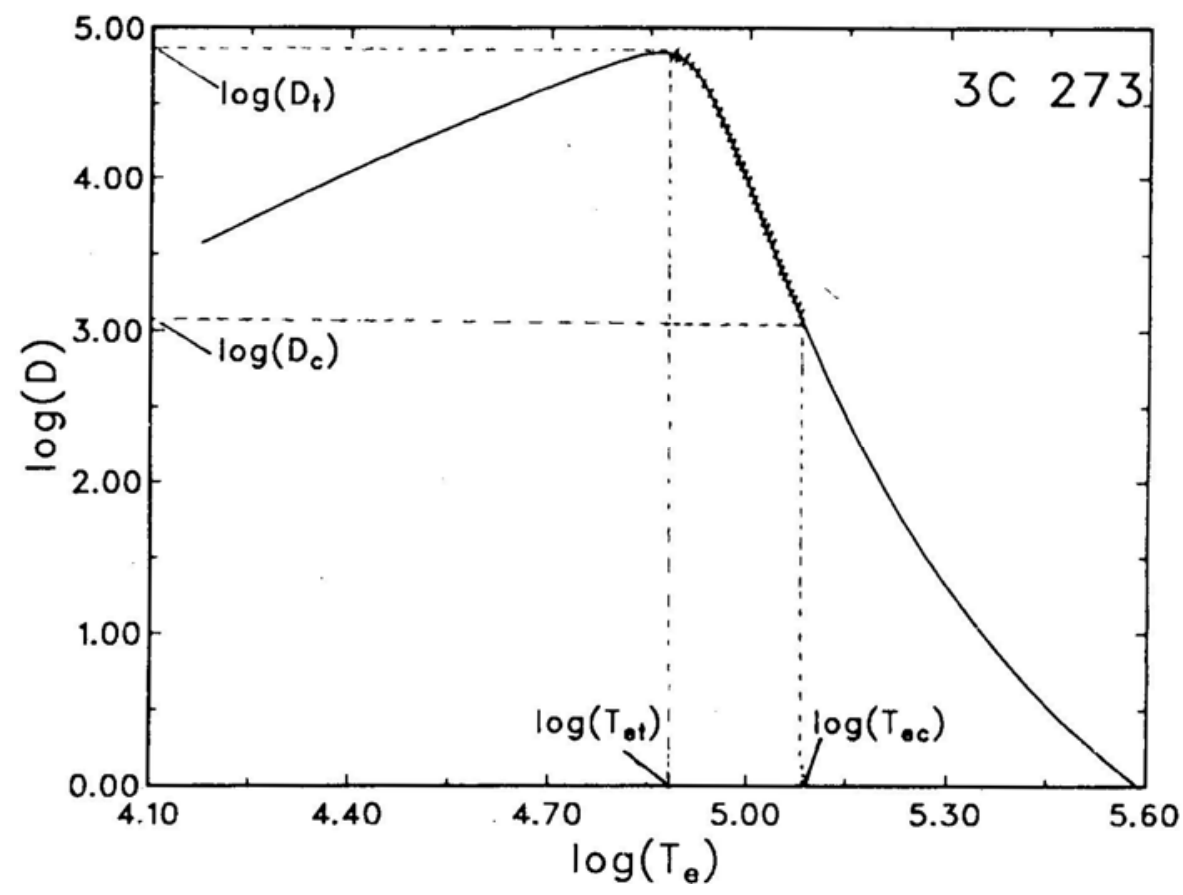

Figure 1. (b) Decay parameter $D$ vs $T_{\mathrm{e}}$ in the 3C 273.

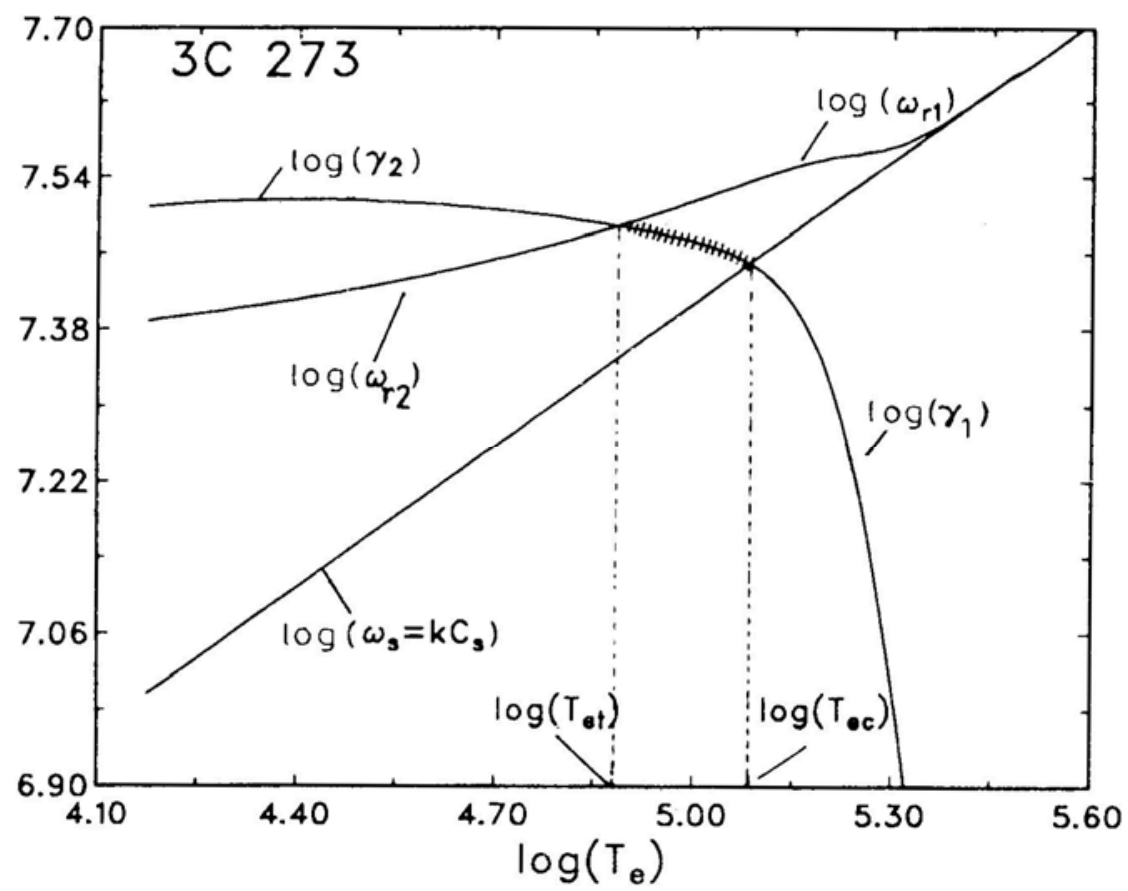

Figure 1. (c) Variation of real of the frequencies of the low frequency daughter mode $\omega_{\mathrm{s}}$, $\omega_{r j}$ and its growth rate $\gamma_{j}(j=1,2) v s T_{\mathrm{e}}$ in the $3 \mathrm{C} 273$. 


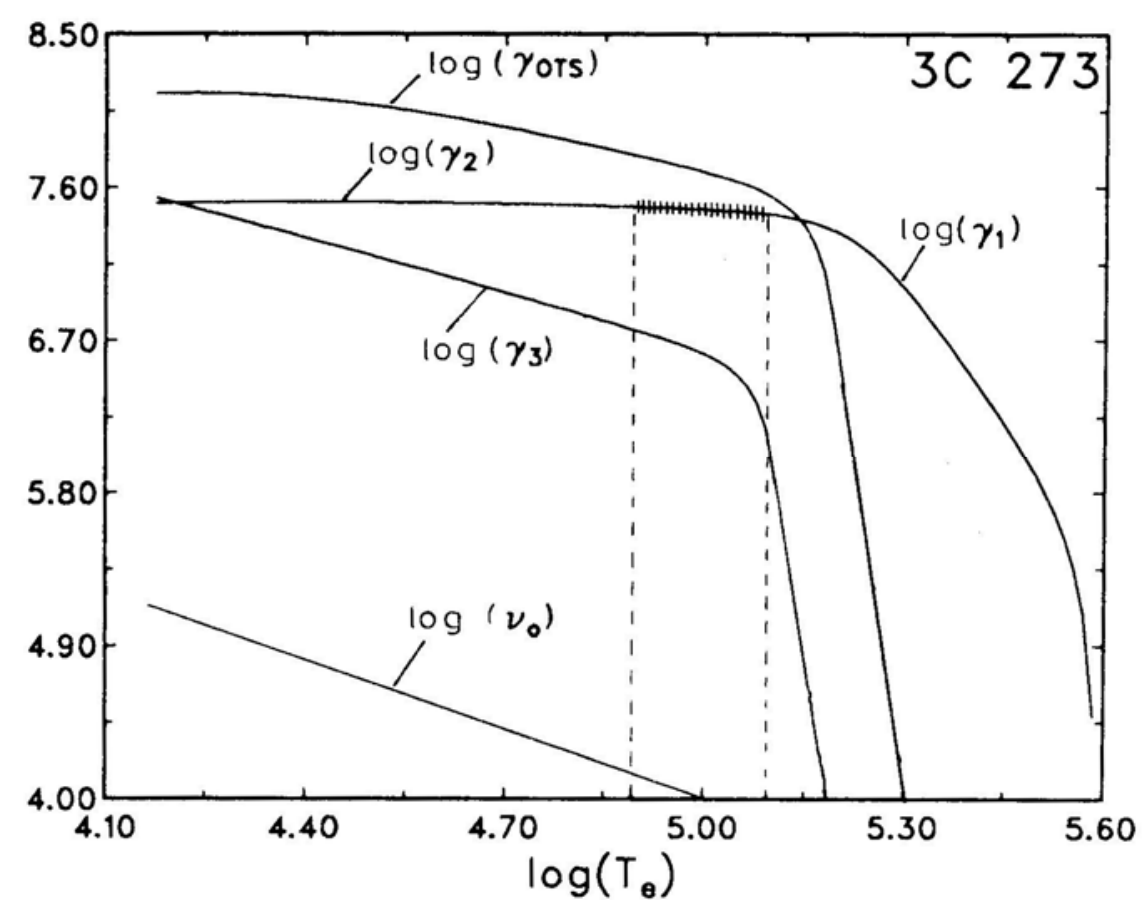

Figure 2. (a) Variation of growth rates $\gamma_{j}(J=1,2,3$ and OTS $) v s T_{\mathrm{e}}$ for a monochromatic pump at $\omega_{0}=1.01 \omega_{\text {pe }}$ in the 3C 273 .

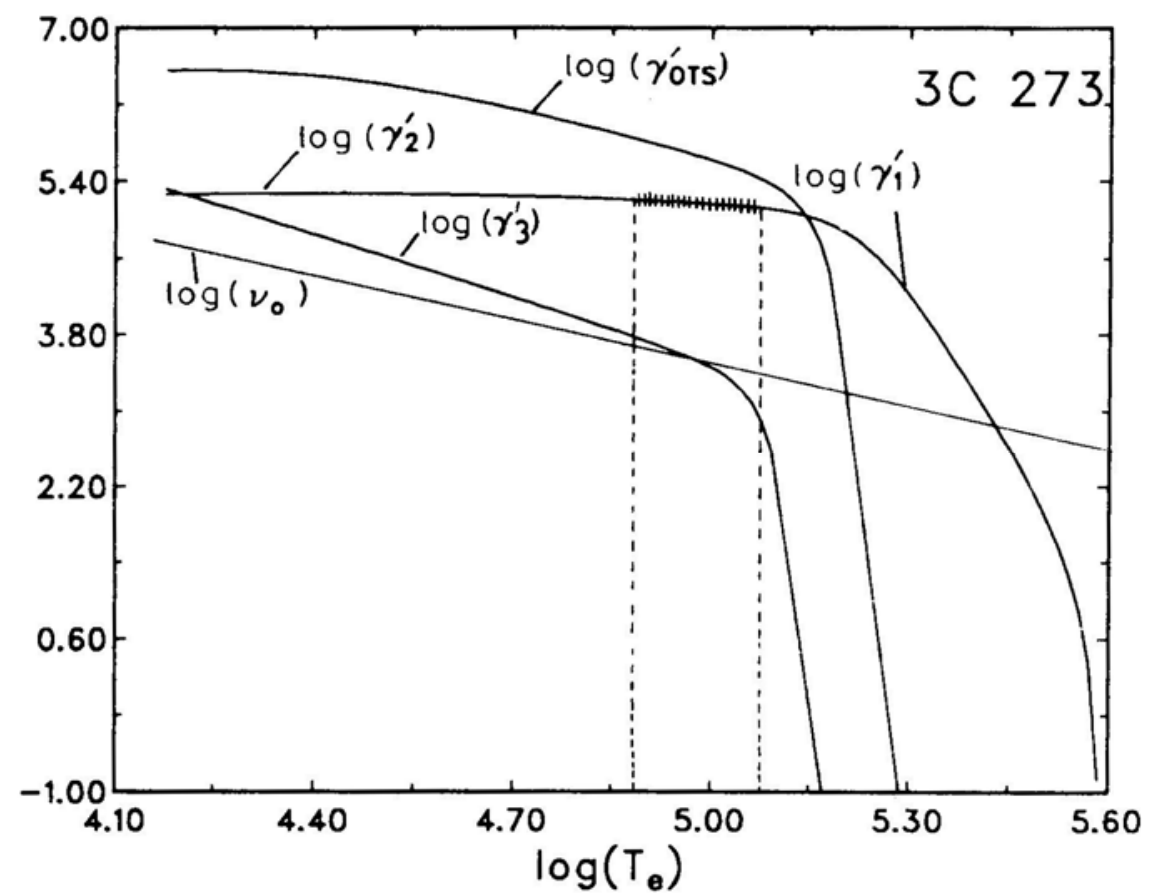

Figure 2. (b) Variation of $\gamma_{j}{ }^{\prime}\left(j=1,2,3\right.$ and OTS) $v s T_{\mathrm{e}}$ using a broad pump in the 3C 273. 


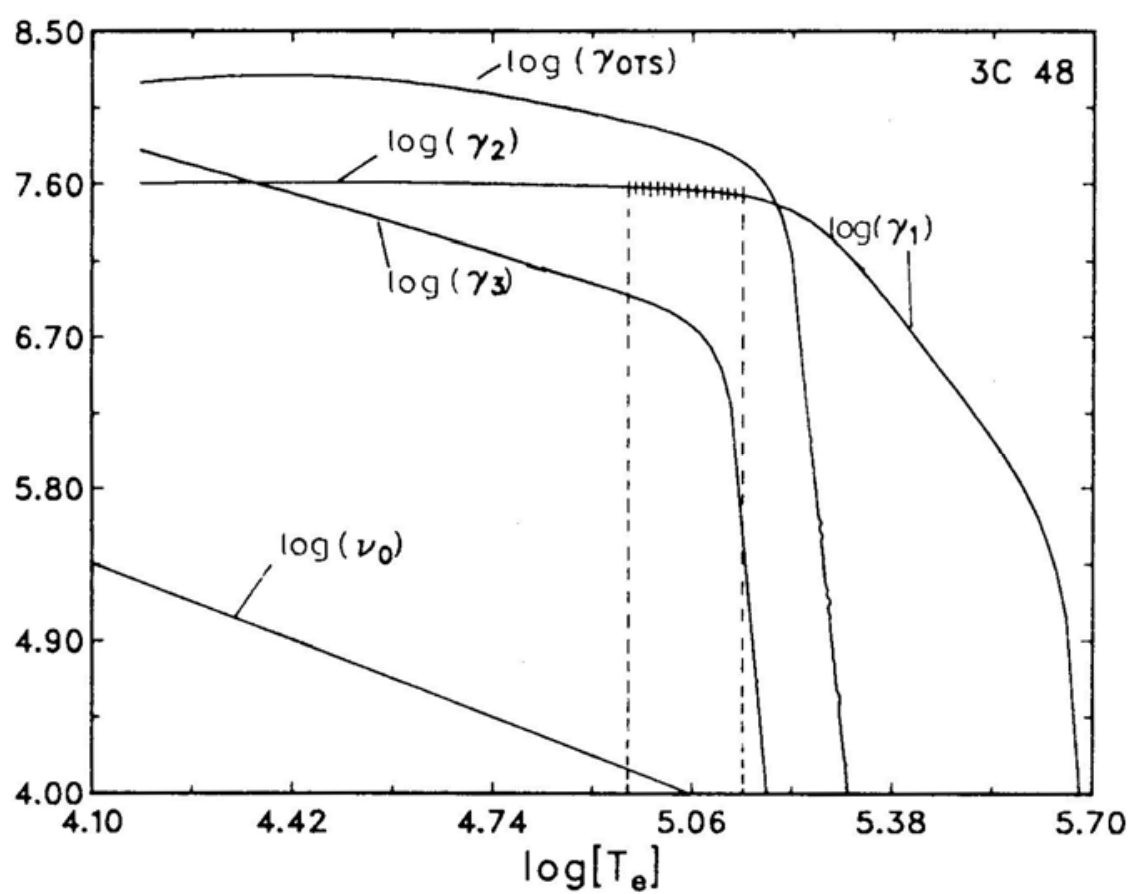

Figure3. (a) Variation of growth rates $\gamma_{j}\left(j=1,2,3\right.$ and OTS) $v s T_{\mathrm{e}}$ for amonochromatic pump at $\omega_{0}=1.01 \omega_{\text {pe }}$ in the $3 \mathrm{C} 48$.

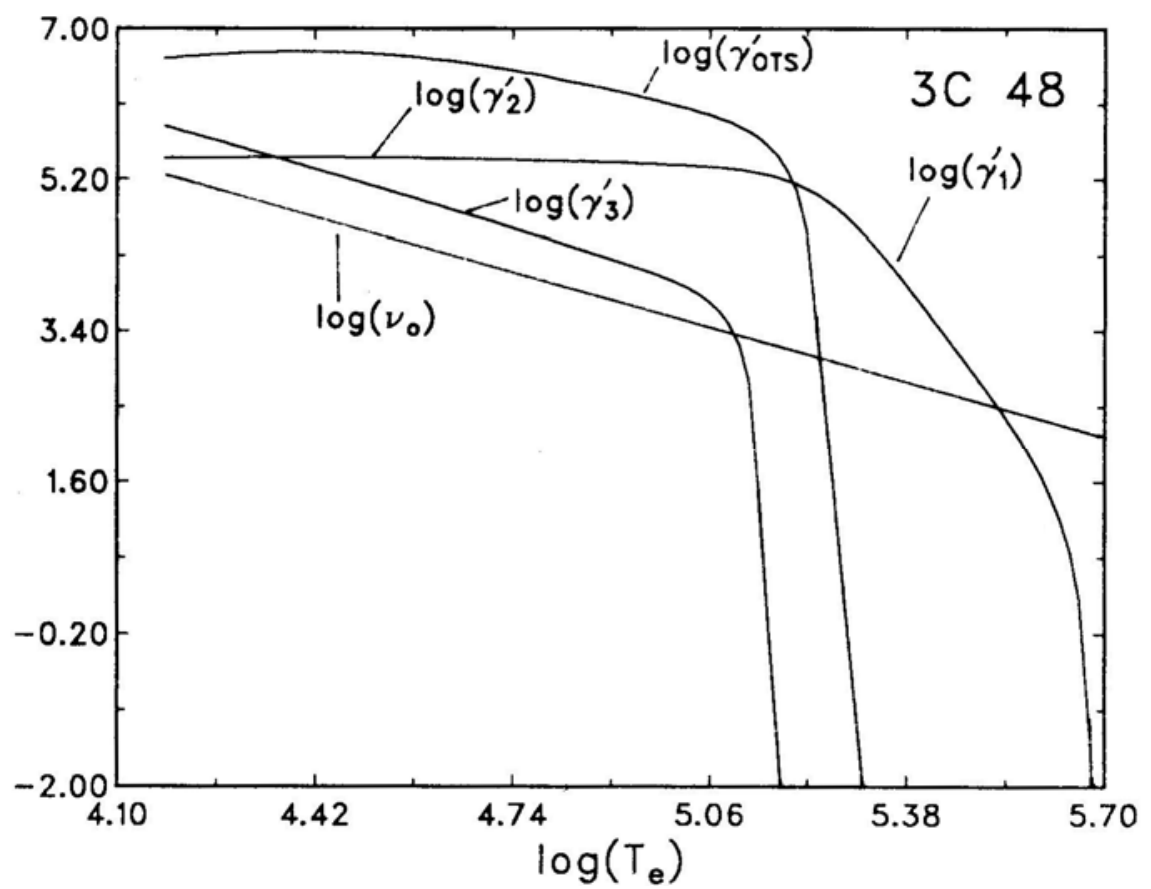

Figure 3. (b) Variation of $\gamma_{j}^{\prime}\left(j=1,2,3\right.$ and OTS) $v s T_{e}$ using a broad pump of width, $\Delta \omega=\omega_{0}$ in the $3 \mathrm{C} 48$. 


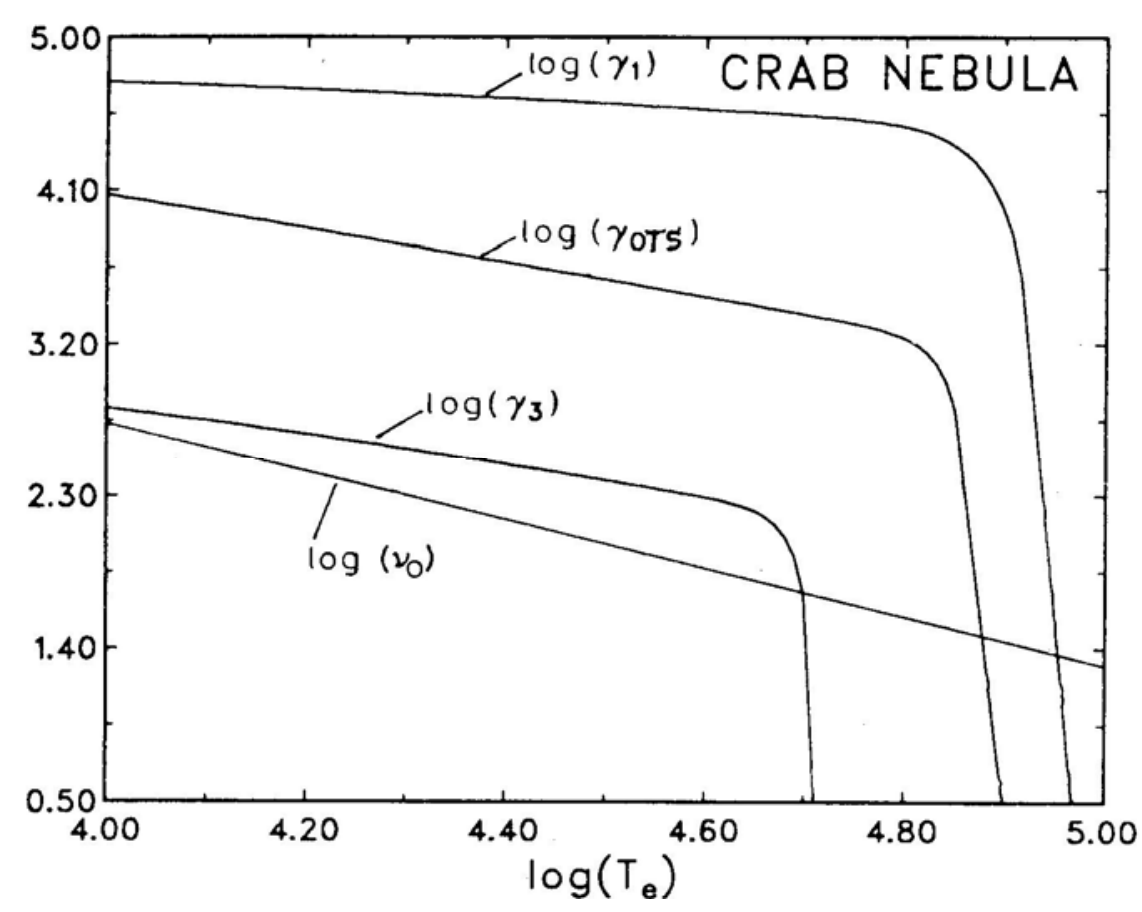

Figure. 4. (a) Variation of growth rates $\gamma_{j}(j=1,3$ and OTS $)$ vs $T_{\mathrm{e}}$ for a monochromatic pump at $\omega=1.01 \omega_{\mathrm{pe}}$ in the Crab Nebula.

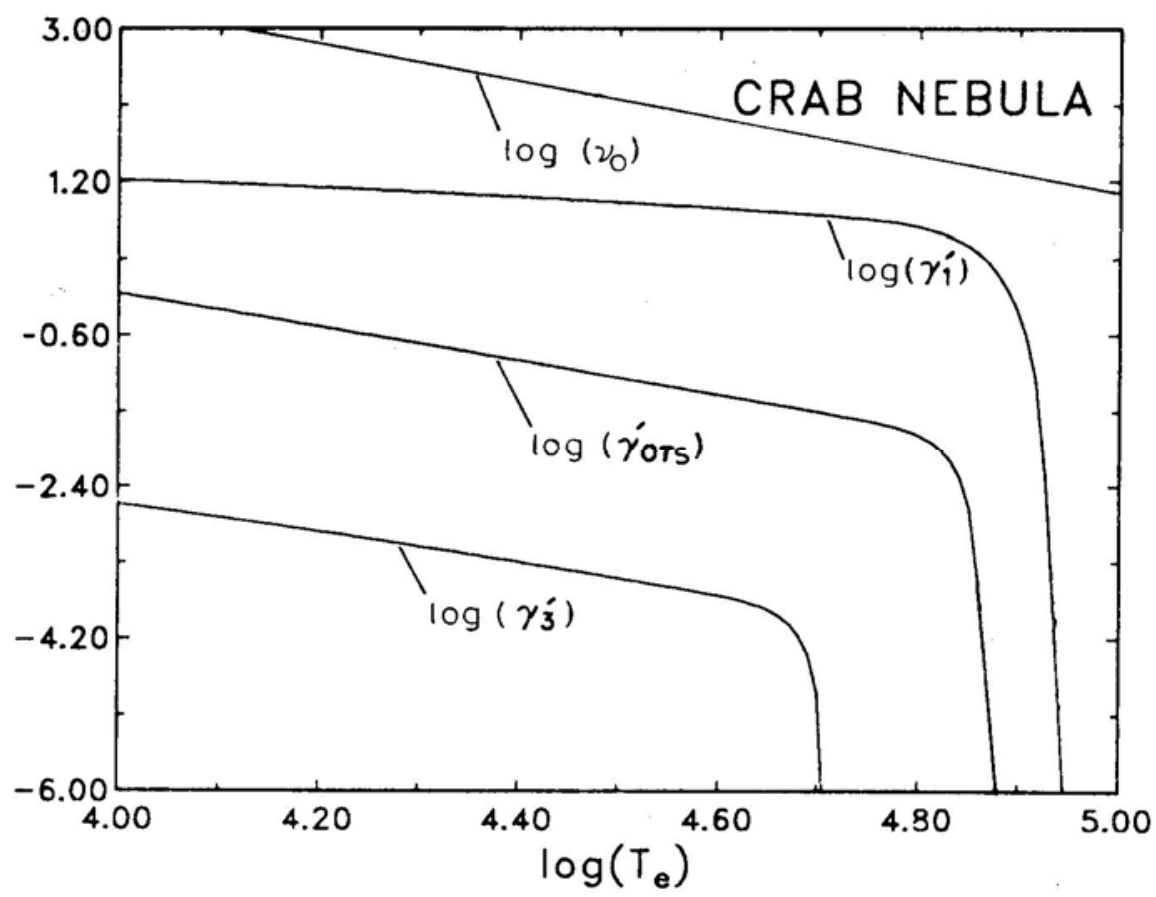

Figure 4. (b) Variation of $\gamma_{j}^{\prime}\left(j=1,3\right.$ and OTS) $v s T_{\mathrm{e}}$ using a broad pump of width $\omega=\omega_{0}$ in the Crab Nebula. Note that PDI-2 is not excited and also collisional absorption is dominant. 


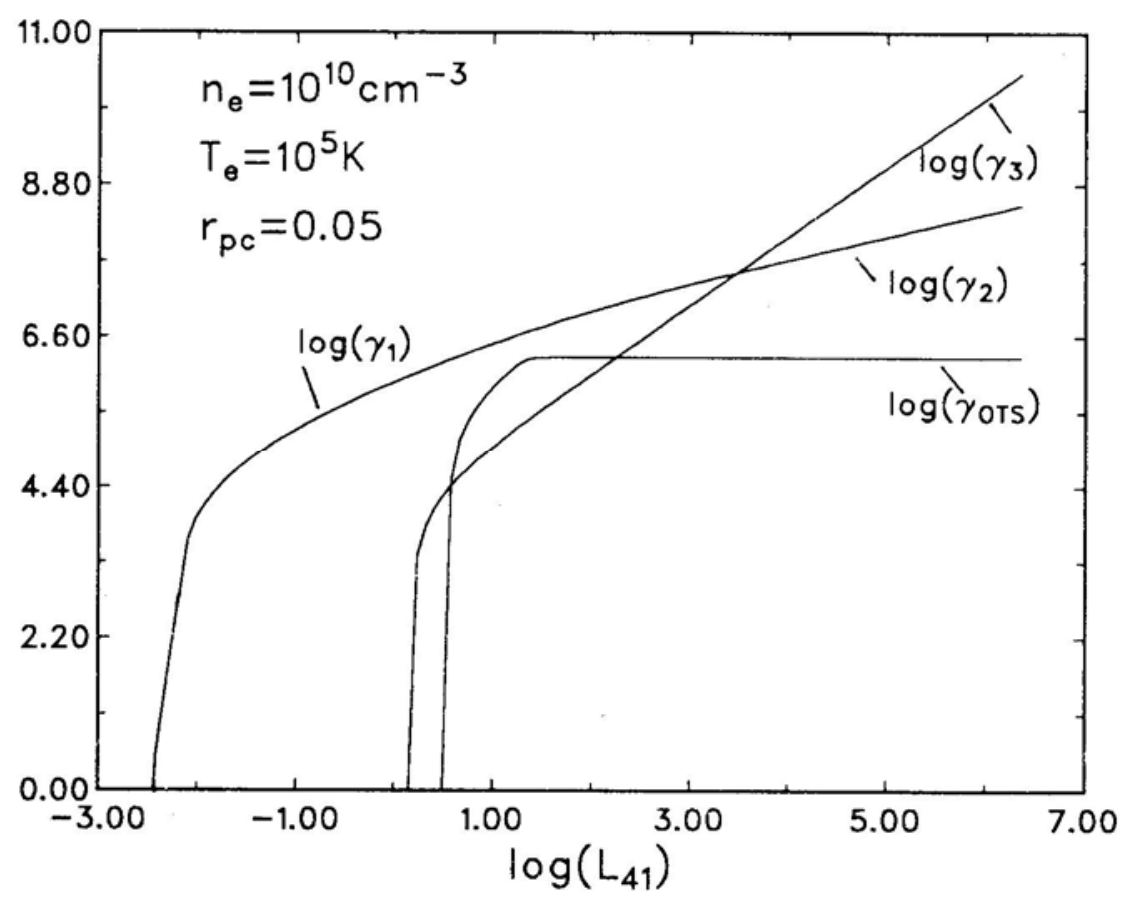

Figure 5. (a) Variation of growth rates $\gamma_{j}=(j=1,2,3$ and OTS $) v s$ pump luminosity $L_{41}$ at $\omega_{0}=1.01 \omega_{\mathrm{pe}}$.

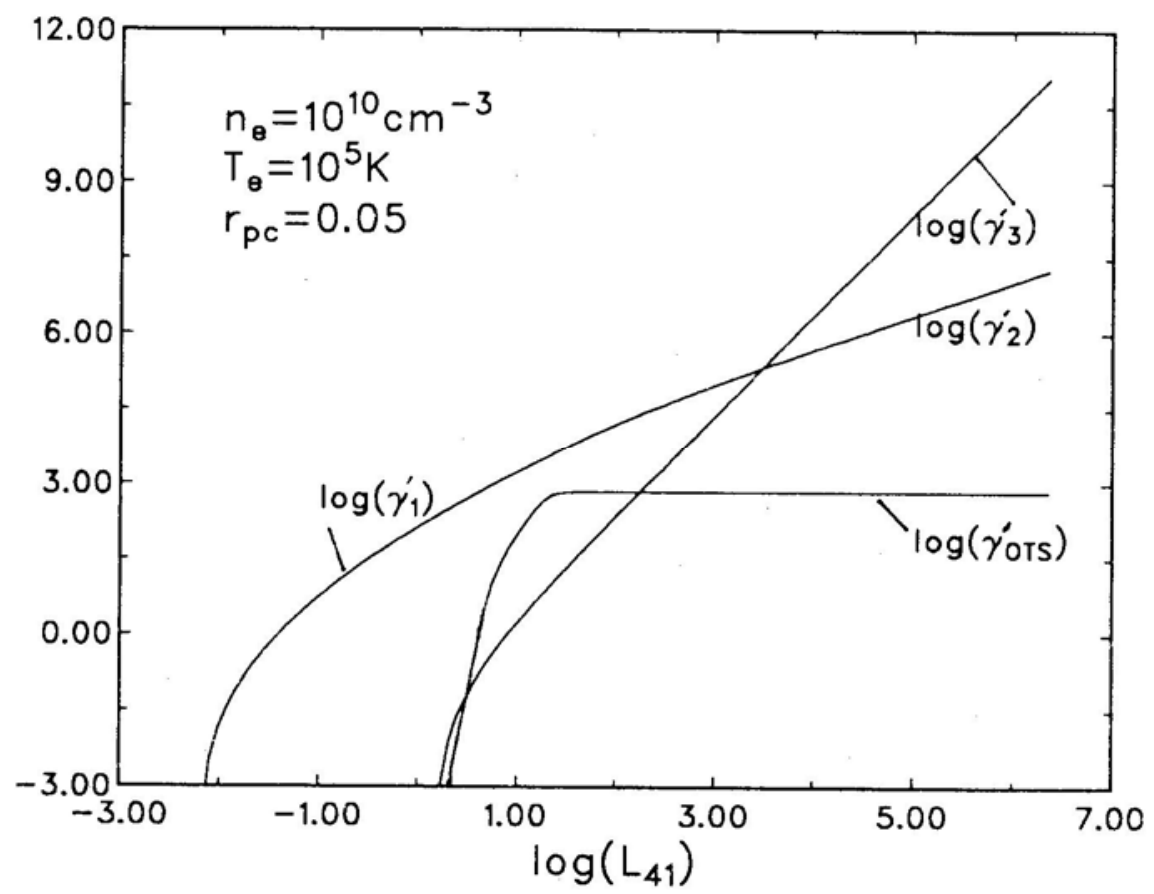

Figure 5. (b) Variation of $\gamma_{j}^{\prime}\left(j=1,2,3\right.$ and OTS) $v s$ luminosity $L_{41}$ of a broad pump of width $\Delta \omega=\omega_{0}$. 


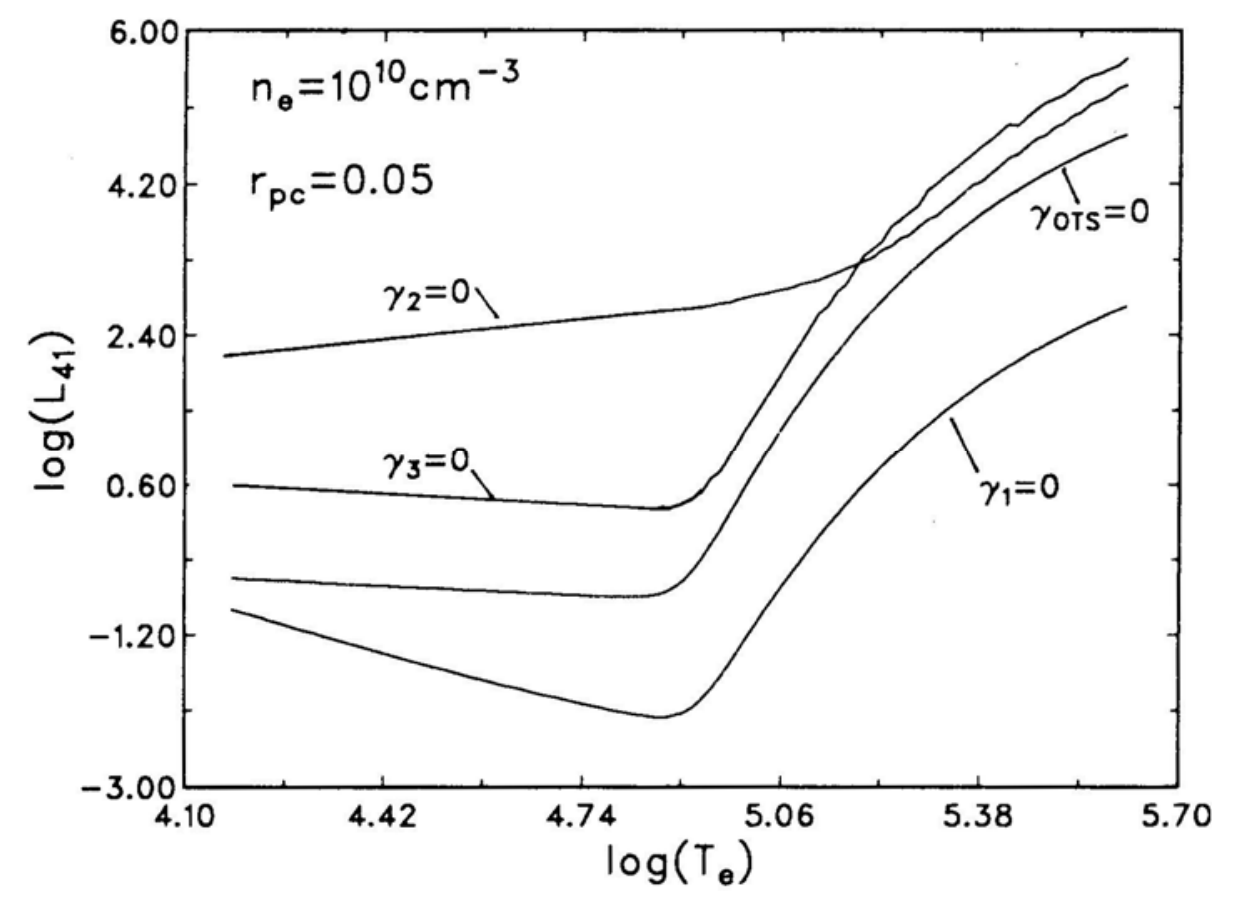

Figure 6. Variation of threshold luminosity $L_{41}$ vs $T_{\mathrm{e}}$ for the plasma parameters shown in the figure. (Fluctuations in curves $\gamma_{\text {отs }}=0$ and $\gamma_{3}=0$ are due to numerical effects)

Table 1. Observed parameters in the resonant interacting region.

\begin{tabular}{lccccc}
\hline Source & $\begin{array}{c}n_{\mathrm{e}} \\
\left(\mathrm{cm}^{-3}\right)\end{array}$ & $\begin{array}{c}\text { minimum } T_{\mathrm{c}} \\
(\mathrm{K})\end{array}$ & $\begin{array}{c}r \\
(\mathrm{pc})\end{array}$ & $\begin{array}{c}\omega_{0} \\
\left(\mathrm{rad} \mathrm{s}^{-1}\right)\end{array}$ & $L_{41}\left(\Delta \omega=\omega_{0}\right)$ \\
\hline 3C 273 & $10^{10}$ & $1.5 \times 10^{4}$ & 0.05 & $8.8 \times 10^{9}$ & $4.59 \times 10^{2}$ \\
3C 48 & $10^{10}$ & $1.5 \times 10^{4}$ & 0.05 & $8.8 \times 10^{9}$ & $8.33 \times 10^{2}$ \\
Crab Nebula & $10^{7}$ & $1.0 \times 10^{4}$ & 0.03 & $1.8 \times 10^{8}$ & $1.20 \times 10^{-4}$ \\
\hline
\end{tabular}

represents PDI- 1 where $\omega_{\mathrm{r} 1} \simeq k c_{s}$ and $\gamma_{1} \ll \omega_{\mathrm{r} 1}$. The variation of growth rate $\gamma_{1}$ of PDI1 with respect to $T_{\mathrm{e}}$ due to a monochromatic pump is shown in Figs 2(a) for 3C 273, 3(a) for 3C 48 and 4(a) for Crab Nebula. Similarly, the variation of growth rate $\gamma_{1}^{\prime}$ of PDI-1 with respect to $T_{\mathrm{e}}$ due to a broad pump of width $\Delta \omega=\omega_{0}$ is shown in Figs 2 (b) for $3 \mathrm{C}$ 273, 3(b) for 3C 48 and 4(b) for Crab Nebula. Fig. 2(a) shows that the maximum electron temperature $T_{e}$ attainable through PDI- 1 is $\simeq 10^{5.6} \mathrm{~K}$. The maximum $T \mathrm{e}$ attained in the $3 \mathrm{C} 48$ is $\simeq 10^{5.7} \mathrm{~K}$ and in the Crab Nebula is $\simeq 10^{5} \mathrm{~K}$ for the values of the parameters indicated in Tables 1 and 2 .

The dependence of the growth rate $y_{1}$ on monochromatic pump strength is shown in the Fig. 5(a). Similarly, Fig. 5(b) shows the variation of $\gamma_{1}^{\prime}$ with respect to the strength of a broad pump. Fig 5(a) shows that at just above the threshold (i.e. when $\gamma_{1}<\Gamma_{\mathrm{i}}$ ), $\gamma_{1}$ is proportional to $\left(L_{41} / r_{\mathrm{pc}}^{2}\right)$ and at well above threshold (i.e. when $\left.\gamma_{1}>\Gamma_{\mathrm{i}}\right)$ it is 
proportional to $\left(L_{41} / r_{\mathrm{pC}}^{2}\right)^{1 / 2}$. Fig. 6 shows the dependence of threshold luminosity on electron temperature $T_{e}$ for all cases of PDI together with OTS. The curve $\gamma_{1}=0$, in Fig. 6, shows the dependence of threshold luminosity of PDI-1 on $T_{e}$. At $T_{\mathrm{e}}<10^{4.5} \mathrm{~K}$ the collisional damping operates and as the temperature goes up Landau damping takes over for $T_{\mathrm{e}}>10^{5.1} \mathrm{~K}$. But in the range $10^{4.5} \mathrm{~K}<T_{\mathrm{e}}<10^{5.1} \mathrm{~K}$ both collisional and Landau damping are small and therefore lower threshold luminosity is needed. The cross marked region in figures represents transition from PDI-1 to PDI-2.

\subsection{PDI-2: Decay into a Reactive Quasi-Ion Mode $\left(\omega_{\mathrm{i} 2}\right)$}

For large pump strength and low temperature, the ion mode loses its linearity. We call this ion mode as the reactive quasi-ion mode because its growth rate $\gamma_{2}$ and frequency $\omega_{\tau 2}$ are much larger than the ion-acoustic wave frequency $\left(\omega_{\mathrm{s}}=k c_{\mathrm{s}}\right)$.

Here $\omega_{0}=\omega_{\mathrm{e}}+\omega_{\mathrm{i} 2}$ and $\omega=\omega_{\mathrm{i} 2}=\omega_{\mathrm{r} 2}+i \gamma_{2}$. The variation of $\omega_{i 2}$ and $y_{2}$ with respect to $T_{\mathrm{e}}$ are shown in the Fig. 1(c) where we define $T_{\mathrm{e}}=T_{\mathrm{ec}}$ and correspondingly $D=D_{c}$ (Fig. lb) at the point where $\gamma_{1}$ crosses $\omega_{\mathrm{s}}$. At this point the ion-acoustic mode loses its linearity. For $T_{\mathrm{e}}<T_{\mathrm{ec}}$ the imaginary part of $\omega$ crosses its real part at $T_{\mathrm{e}}=T_{\mathrm{et}}$ and $D=D_{\mathrm{t}}$ (Fig. lb). In Fig. 1(c) the range $T_{\mathrm{e}}<T_{\text {et }}$ represents the region of reactive quasi-ion mode. The range $T_{\mathrm{et}} \leqslant T_{\mathrm{e}} \leqslant T_{\mathrm{ec}}$ and $D_{\mathrm{t}} \geqslant D \geqslant D_{e}$ represents the transition region in which the reactive quasi-ion mode changes into an ion-acoustic mode or vice versa. Figs 1 (b) and 1(c) shows for 3C 273, $T_{\text {ec }}=10^{5.08} \mathrm{~K}, T_{\text {et }}=10^{4.89} \mathrm{~K}, D_{\mathrm{c}}=10^{3.2}$ and $D_{\mathrm{t}}=10^{4.8}$. Under the coherent pump approximation, the variation of growth rate $\gamma_{2}$ with respect to $T_{\mathrm{e}}$ is shown in Figs 2(a) for 3C 273 and 3(a) for 3C 48. Similarly, using a broad pump of width $\Delta \omega=\omega_{0}$, the variation of growth rate $\gamma_{2}^{\prime}$ with respect to $T_{\mathrm{e}}$ is shown in Figs 2(b) for 3C 273 and 3(b) for 3C 48.

For $T_{\mathrm{e}}<T_{\mathrm{et}}$ and $\mathrm{D}>D_{\mathrm{t}}, \gamma_{2} / \omega_{\mathrm{r} 2}=\sqrt{3}$. The variation of growth rate $\gamma_{2}$ with respect to the luminosity of the pump is shown in the Fig. 5(a). We find that the $\gamma_{2}$ is proportional to $\left(L_{41} / r_{c}^{2}\right)$ Similarly, the variation of growth rate $\gamma_{2}^{\prime}$ with the luminosity of a broad pump is shown in Fig. 5(b). The curve $\gamma_{2}=0$ in the Fig. 6 shows the variation of the threshold luminosity with respect to $T_{\mathrm{e}}$. This mode excites only when $D>D_{\mathrm{c}}$ or $L_{41}>4 \pi R^{2} n_{\mathrm{e}} c / P$. We find $L_{41}>10^{2 \cdot 38}$ at $T_{\mathrm{ec}}=10^{5.08} \mathrm{~K}$ for $3 \mathrm{C} 273$. This mode is not excited in the Crab Nebula because the radio luminosity is too low to cross the threshold luminosity $L_{41}=1.1 \times 10^{2}$, calculated using parameters given in Tables 1 and 2. We find that PDI2 occurs at the lower temperature $T_{\mathrm{e}}<T_{\mathrm{ec}}$ and the collisional damping rate $v_{0}$ of the pump is still much smaller than the growth rate $\gamma_{2}$ of a reactive quasi-ion mode.

Table 2. Model parameters.

\begin{tabular}{lcc}
\hline $\begin{array}{l}\text { Type of } \\
\text { instability }\end{array}$ & $\begin{array}{c}\text { minimum } \\
(\mathrm{K})\end{array}$ & $\begin{array}{c}\omega_{0} \\
\left(\mathrm{rad} \mathrm{s}^{-1}\right)\end{array}$ \\
\hline PDI-1 & $T_{\mathrm{e}} / 100$ & $1.01 \omega_{\mathrm{pe}}$ \\
PDI-2 & $T_{\mathrm{e}} / 100$ & $1.01 \omega_{\mathrm{pe}}$ \\
PDI-3 & $10 \times T_{\mathrm{e}}$ & $1.01 \omega_{\mathrm{pe}}$ \\
OTS & $T_{\mathrm{e}} / 100$ & $\left(\omega_{\mathrm{e}}+\omega_{\mathrm{pe}}\right) / 2$ \\
\hline
\end{tabular}




\subsection{PDI-3: Decay into a Resistive Quasi-Ion Mode $\left(\omega_{\mathrm{i} 3}\right)$}

Here $\omega_{0}=\omega_{\mathrm{e}}+\omega_{13}$. For $T_{\mathrm{e}} \leqslant T_{\mathrm{i}}$, the phase velocity of the ion-acoustic mode $\left(c_{\mathrm{s}}\right)$ becomes slightly greater than thermal velocity $\left(v_{\mathrm{i}}\right)$ of the ions. Therefore, the beat mode (i.e. ion mode) of a pump wave and an electron plasma mode undergoes the nonlinear Landau damping over the thermal ions. Since the ion mode is strongly damped, a weak instability is excited compared to other three cases.

From Equation (3), for $\omega=\omega_{13}=\omega_{0}+i \gamma_{3}$, we get

$$
\begin{gathered}
\omega_{\mathrm{r} 3}=\left.\frac{\left(\mathbf{K} \cdot \mathbf{V}_{0}\right)^{2}}{2 \omega_{\mathrm{pe}}} \operatorname{Re}\left\{\frac{\chi_{\mathrm{e}}\left(\chi_{\mathrm{i}}+1\right)}{\varepsilon(K, \omega)}\right\}\right|_{\frac{\omega}{k}=\frac{\Delta}{k}}+\Delta \\
\gamma_{3}=\left.\frac{\left(\mathbf{K} \cdot \mathbf{V}_{0}\right)^{2}}{2 \omega_{\mathrm{pe}}} \operatorname{Im}\left\{\frac{\chi_{\mathrm{e}}\left(\chi_{\mathrm{i}}+1\right)}{\varepsilon(K, \omega)}\right\}\right|_{\substack{\omega=\Delta \\
k \\
k}}+\Delta .
\end{gathered}
$$

Using a coherent pump, the variation of $\gamma_{3}$ with respect to $T_{\mathrm{e}}$ is shown in Figs 2(a) for 3C 273, 3(a) for 3C 48, and 4(a) for Crab Nebula. Similarly, using a broad pump having the width $\Delta \omega=\omega_{0}$, we have plotted y 3 with respect to $T_{\mathrm{e}}$ in Figs 2(b) for $3 \mathrm{C} 273,3$ (b) for $3 \mathrm{C} 48$ and 4(b) for Crab Nebula. We find $\omega_{\mathrm{r} 3} \simeq \mathrm{A}=\omega_{\mathrm{s}}$ and $\gamma_{3}$ is small compared to $\omega_{\mathrm{r} 3}$, $\gamma_{1}, \gamma_{2}$ and $\gamma_{\text {OTs. }}$.

In the BLR of 3C 273 and 3C 48 the collisional damping rate $v_{0}$ of the pump wave is smaller than $\gamma_{3}$ but it is approximately equal to $\gamma_{3}$ in Crab Nebula. Since width chosen is large, the figure shows that growth rates of all the cases of PDT are smaller than $v_{0}$.

The dependence of $\gamma_{3}$ on the monochromatic pump strength is shown in the Fig. 5(a). We find $y$, is proportional to $\left(L_{41} / r_{\mathrm{pc}}^{2}\right)$. Similarly, the dependence of $\gamma_{3}^{\prime}$ on the broad pump strength is shown in the Fig. 5(b). The curve $\gamma_{3}=0$ in Fig. 6 shows the dependence of threshold luminosity on Te.

\subsection{Oscillating Two-Stream Instability (OTS)}

This instability excites when an intense e.m. pump of frequency less than the frequency of electron plasma wave interacts with the plasma. Here $\omega_{\mathrm{pe}}<\omega_{0}<\omega_{\mathrm{e}}$. The pump e.m. wave $\left(\omega_{0}, \mathbf{K}_{0}\right)$ couples with a nigh frequency wave $\left(\omega_{\mathrm{e}}, \mathbf{K}_{\mathrm{e}}\right)$ and a non-oscillatory ion mode $\left(0, \mathbf{K}_{\mathrm{i}}\right)$. We find that $\mathbf{K}_{\mathrm{e}} \simeq-\mathbf{K}_{\mathrm{i}} \equiv \mathbf{K}$. In this case the thermal ion density fluctuations of zero frequency grow along with an electron plasma mode. Since these static ion density fluctuations do not qualify for a normal mode of the plasma, we can not include OTS in the class of parametric decay instabilities. However, it has been considered as a special case of the parametric instability.

Here both the terms $\varepsilon\left(\mathbf{K}, \omega-\omega_{0}\right)$ and $\varepsilon\left(\mathbf{K}, \omega+\omega_{0}\right)$ are resonant. For $\omega=0+i \gamma_{\mathrm{OTS}}$, Equation (3) gives

$$
\frac{1}{1-\frac{\sqrt{\pi^{\gamma \text { OTs }}}}{k v_{\mathrm{e}}}}+\frac{1}{\left(1-\frac{\sqrt{\pi} \gamma_{\mathrm{OTS}}}{k v_{\mathrm{i}}}\right) \frac{T_{\mathrm{e}}}{T_{\mathrm{i}}}+\left(k \lambda_{\mathrm{De}}\right)^{2}}=-\frac{\left(\mathbf{K} \cdot \mathbf{V}_{\mathrm{o}}\right)^{2} \Delta}{\left(k \lambda_{\mathrm{De}}\right)^{2} \omega_{\mathrm{pe}}\left\{\left(\gamma_{\mathrm{OTS}}+\Gamma_{\mathrm{e}}\right)^{2}+\Delta^{2}\right\}} .
$$


If we simplify Equation (13) for $\gamma_{\mathrm{OTS}}$, we get

$$
a_{1} \gamma_{\mathrm{OTS}}^{3}+a_{2} \gamma_{\mathrm{OTS}}^{2}+a_{3} \gamma_{\mathrm{OTS}}+a_{4}=0 \text {, }
$$

where

$$
\begin{aligned}
a_{1}= & \frac{\sqrt{\pi}}{k v_{\mathrm{e}}}+\frac{\sqrt{\pi}}{k v_{\mathrm{i}}} \frac{T_{\mathrm{e}}}{T_{\mathrm{i}}}, \\
a_{2}= & 2 \Gamma_{\mathrm{e}} a_{1}-\left(k \lambda_{\mathrm{De}}\right)^{2}-\frac{T_{\mathrm{e}}}{T_{\mathrm{i}}}-\frac{\left(\mathbf{K} \cdot \mathbf{V}_{0}\right)^{2}}{\omega_{\mathrm{pe}}\left(k \lambda_{\mathrm{De}}\right)^{2}} \frac{\pi \Delta}{v_{\mathrm{e}} v_{\mathrm{i}} k^{2}} \frac{T_{\mathrm{e}}}{T_{\mathrm{i}}}-1, \\
a_{3}= & a_{1}\left(\Gamma_{\mathrm{e}}^{2}+\Delta^{2}\right)-2\left(1+\left(k \lambda_{\mathrm{De}}\right)^{2}+\frac{T_{\mathrm{e}}}{T_{\mathrm{i}}}\right) \Gamma_{\mathrm{e}} \\
& +\frac{\left(\mathbf{K} \cdot \mathbf{V}_{0}\right)^{2} \Delta}{\omega_{\mathrm{pe}}\left(k \lambda_{\mathrm{De}}\right)^{2}}\left\{\left(\frac{\sqrt{\pi}}{k v_{\mathrm{e}}}+\frac{\sqrt{\pi}}{k v_{\mathrm{i}}}\right) \frac{T_{\mathrm{e}}}{T_{\mathrm{i}}}+\frac{\sqrt{\pi}\left(k \lambda_{\mathrm{De}}\right)^{2}}{k v_{\mathrm{e}}}\right\}
\end{aligned}
$$

and

$$
a_{4}=-\left(1+\left(k \lambda_{\mathrm{De}}\right)^{2}+\frac{T_{\mathrm{e}}}{T_{\mathrm{i}}}\right)\left(\Gamma_{\mathrm{e}}^{2}+\Delta^{2}\right)-\frac{\left(\mathbf{K} \cdot \mathbf{V}_{\mathrm{o}}\right)^{2} \Delta}{\omega_{\mathrm{pe}}\left(k \lambda_{\mathrm{De}}\right)^{2}}\left(\left(k \lambda_{\mathrm{De}}\right)^{2}+\frac{T_{\mathrm{e}}}{T_{\mathrm{i}}}\right) .
$$

For $k \lambda_{\mathrm{De}} \simeq 0.4$, Equation (13) gives

$$
\gamma_{\mathrm{OTS}}= \pm i\left[\frac{\left(\mathbf{K} \cdot \mathbf{V}_{0}\right)^{2}}{\left(k \lambda_{\mathrm{De}}\right)^{2} \omega_{\mathrm{pe}}} \Delta\left\{1+\frac{1}{\left(k \hat{\lambda}_{\mathrm{De}}\right)^{2}+\frac{T_{\mathrm{e}}}{T_{\mathrm{i}}}}\right\}^{-1}+\Delta^{2}\right]^{1 / 2}-\Gamma_{\mathrm{e}}
$$

The $\gamma_{\mathrm{OTS}}$ is real only when $\Delta=\omega_{0}-\omega_{\mathrm{e}}<0$, i.e. $\omega_{0}<\omega_{\mathrm{e}}$. The expression $\gamma_{\mathrm{OTS}}=0$ gives $\Delta$ $=\Delta_{\text {thr }}$ while $\frac{\partial \gamma_{\text {oTs }}}{\partial \Delta}=0$ gives $\Delta=\Delta_{\max }$ corresponding to a maximum growth rate. Now, $\Delta_{\text {thr }}=\Delta_{\max }$ gives the minimum pump strength at which instability occurs:

$$
\left(\frac{v_{0}}{v_{\mathrm{e}}}\right)^{2}=2\left\{1+\frac{1}{\left(k \lambda_{\mathrm{De}}\right)^{2}+\frac{T_{\mathrm{e}}}{T_{\mathrm{i}}}}\right\} \frac{\Gamma_{\mathrm{e}}}{\omega_{\mathrm{pe}}} .
$$

We solve Equation (14), using $k=8.75 \mathrm{~cm}^{-1}$ for 3C 273 and $3 \mathrm{C} 48$, and $k=6.0 \mathrm{~cm}^{-1}$ for Crab Nebula where $k$ has been determined using the condition $\omega_{\mathrm{pe}}<\omega_{0}<\omega_{\mathrm{e}}$ and the values of parameters given in the Tables 1 and 2 . One of the three roots represents a growing electron mode and an ion density fluctuation while the other two represent damped modes. Using $\Delta=\Delta_{\max }$ and a monochromatic pump, the growth rate $\gamma_{\text {OTS }}$ is plotted as a function of $T_{\mathrm{e}}$ in Figs 2(a) for 3C 273, 3(a) for 3C 48 and 4(a) for Crab Nebula. Similarly, using a broad pump, $\gamma_{\text {OTs }}^{\prime}$ is plotted as a function of $T_{\mathrm{e}}$ in Figs 2 (b) for 3C 273, 3(b) for 3C 48 and 4(b) for Crab Nebula. The dependence of $\gamma_{\text {OTs }}$ on a monochromatic pump luminosity is shown in the Fig. 5(a). The dependence of $\gamma_{\text {OTS }}^{\prime}$ on the broad pump luminosity is shown in the Fig. 5(b): The curve $\gamma_{\mathrm{OTS}}=0$ in the Fig. 6 shows the variation of threshold luminosity with $T_{e}$. Again one notices that the $\gamma_{\text {OTS }}$ is much larger than the collisional absorption rate $v_{0}$, of e.m. pump wave. 


\section{Formation of $21 \mathrm{~cm}$ absorption line in the emission-line region}

The 21-cm absorption line has been observed from several QSO systems. It is believed to originate in the neutral hydrogen clouds in the vicinity of the QSO. This absorption line can also originate when the radiation of frequency $1420 \mathrm{MHz}(21-\mathrm{cm})$ drives the PDI in the plasma having electron plasma frequency close to $1420 \mathrm{MHz}$. This effect was first considered by Krishan (1988). This process has line character in principle since it occurs only when a frequency matching condition is satisfied. The presence of $21 \mathrm{~cm}$ absorption line indicates that the electron density in the emission-line region must be $\simeq 2.5 \times 10^{10} \mathrm{~cm}^{-3}$, which falls within the range of inferred densities for these regions. The $21-\mathrm{cm}$ radiation luminosities in $3 \mathrm{C} 273$ and $3 \mathrm{C} 48$ are sufficient to drive PDI.

For comparison, the standard expression (Krishan 1988) for absorption rate for the spin flip transition is given by

$$
K(\omega)=2.58 \times 10^{-15} \frac{f(\omega) N}{T_{\mathrm{K}}} \mathrm{cm}^{-1}
$$

where $T_{\mathrm{K}}$ is the temperature which characterizes the population distribution between the two atomic states, $f(\omega)$ is the line shape function normalized to unity and $N$ is the number of hydrogen atoms $\mathrm{cm}^{-3}$. Using some standard values of the different parameters we find absorption rate due to PDI in the ionized region is much larger than the absorption rate for spin flip transition in the neutral hydrogen region.

\section{Results and conclusions}

(1) An incident e.m. pump wave drives purely electrostatic oscillations at two frequencies close to $\omega_{\mathrm{pe}}$ and $\omega_{\mathrm{pi}}$.

(2) A parametric amplification occurs for drive frequencies $\omega_{0} \geqslant \omega_{\rho \mathrm{\rho e}}$ and for driver amplitude exceeding a certain critical threshold.

(3) Instability is maximum when $\mathbf{K} \| \mathbf{E}_{0}$ and zero for $\mathbf{K} \perp \mathbf{E}_{0}$.

(4) For circularly polarized pumps the general picture remains unchanged, since growth rates are again determined by the component of $\mathbf{K}_{0}$ parallel to $\mathbf{K}$.

(5) The absorption rate of e.m. wave depends on the ratio $\left(L_{41} / r_{\mathrm{pc}}^{2}\right)$ and it is much larger than the free-free absorption rate $v_{0}$.

(6) Electron temperature $T_{\mathrm{e}}$ and ion temperature $T_{\mathrm{i}}$ increase by multiples of tens for moderate radio luminosities.

Compared to all other processes reviewed by Davidson \& Netzer (1979), PDI is a faster process. The excited Langmuir wave will heat the plasma when it undergoes Landau damping. Apart from damping it can undergo many other processes: $\omega_{\mathrm{e}}+\omega_{\mathrm{e}} \rightarrow \omega_{0}^{\prime}, \omega_{\mathrm{e}} \rightarrow \omega^{\prime} \mathrm{e}+\omega_{\mathrm{i}}^{\prime}$, etc. In the first process, two electron plasma waves combine together to produce an e.m. wave. In the second case, it drives PDI, and produces electron and ion-acoustic waves. Therefore, an incident e.m. energy can heat the plasma medium, as well as get reprocessed to other frequencies.

The growth rates indicate the rates at which the plasma medium and the radio radiation try to attain equilibrium with each other. The threshold condition (Equation (9), Fig. 6) ensures those values of plasma parameters like density and temperature for which the fast process of PDI does not occur and the equilibrium is 
reached. Decay instability may be the mechanism for the formation of hot lower density corona adjoining each photoionized dense region. The dip in the spectrum of 3C 273 at $5.0 \times 10^{9} \mathrm{~Hz}$ (Cowsik \& Lee 1982) may be due to the anomalous absorption of radio waves through PDI. Since PDI is an efficient process, it cannot be ignored while accounting for the observed value of radio luminosity which is less than that obtained by extrapolation from the high frequency part of the spectrum.

\section{Acknowledgment}

Discussions with Prof. Paul J. Wiita during this work are gratefully acknowledged.

\section{References}

Collin-Souffrin, S., Lasota, J. P. 1988, Publ. astr. Soc. Pacific, 100, 1041.

Cowsik, R., Lee, Y. C. 1982, Proc. R. Soc. London Ser. A383, 409.

Davidson, K., Netzer, H. 1979, Rev. Mod. Phys., 51, 715.

Dobrowoniy, M., Ferrori, A. 1976, Astr. Astrophys., 47, 97.

Drake, J. F., Kaw, P. K., Lee, Y. C., Schmidt, G., Liu, C. S., Rosenbluth, M. V. 1974, Phys. Fluids, 17, 778.

Gangadhara, R. T., Krishan, V. 1990, in I AU Symp. 142: Basic Plasma Processes on the Sun, Eds E. R. Priest \& V. Krishan, D. Reidel, Dordrecht (in press).

Gangadhara, R. T., Krishan, V. 1990, in Proc. Atlanta meeting on Variability of Active Galactic Nuclei, Eds D. Miller \& P. J. Wiita, Cambridge Univ. Press, (in press).

Ginzburg, V. L., Ozernoy, L. M. 1976, Astrophy. J., 144, 599.

Krishan, V. 1987, Mon. Not. R. astr. Soc., 226, 629.

Krishan, V. 1988, Mon. Not. R. astr. Soc., 231, 353.

Krolik, J. H., McKee, C. F., Tarter, C. B. 1978, in Proc. Pittsburgh Conf. on BL Lacertae Objects, Ed A. Wolfe, Univ. Pittsburgh. p. 277.

Kruer, W. L. 1988, in The Physics of Laser-Plasma Interactions, Addison-Wesley, New York, p. 70.

Kwan, J., Krolik, J. H. 1981, Astrophys. J., 278, 558.

Lang, K. R. 1980, Astrophysical formulae, Springer-Verlag, Berlin, p. 195.

Liu, C. S., Kaw, P. K. 1976, Advances in plasma physics, 6, 83. 